\title{
Efeito do step-training no aumento da força muscular em mulheres idosas saudáveis
}

\author{
Effect of a step-training program on muscle strength in older \\ women
}

1. Centro Universitário de Belo Horizonte. Departamento de Ciências Biológicas, Ambientais e da Saúde. Laboratório de Biomecânica, Belø Horizonte, MG. Brasil.

2. Universidade Federal de Minas Gerais. Departamento de Esportes. Laboratório de Biomecânica. Belo Horizonte. MG. Brasil.

Recebido em 05/08/2009 Revisado em 17/10/2009 Aprovado em 18/12/2009
Resumo - O step-training é uma atividade física associada com aumento da força de membros inferiores. A força muscular é um componente crítico para a manutenção da capacidade funcional. O objetivo deste estudo foi verificar o efeito de seis semanas de steptraining nos parâmetros trabalho e potência de membros inferiores em mulheres idosas. Treze mulheres saudáveis e ativas se voluntariaram a participar do estudo. Todas foram submetidas a aulas de step-training, três vezes por semana, durante 60 minutos cada. As variáveis da força muscular dos extensores e flexores do joelho foram mensuradas por meio de um dinamômetro isocinético Biodex System 3 Pro. Trabalho e potência muscular foram mensurados nas velocidades angulares de 60 e $180 \%$ s. Diferença significativa $(p<0.05)$ foi encontrada no trabalho para os músculos flexores do joelho, entre o pré e pós-teste, nas velocidades de 60 e 180\%. Houve diferença significativa $(\mathrm{p}<0.05)$ para o trabalho nos músculos extensores na comparação pré e pós-teste, na velocidade angular de $60 \%$ s. Diferenças significativas foram observadas na potência muscular na comparação pré e pós-teste, para os músculos flexores na velocidade angular de $60 \%$ ( p < 0,05) e para os músculos extensores do joelho nas velocidades angulares de 60 e 180\% $(p<0,05)$. Concluindo, o step-training pode ser recomendado como uma atividade física alternativa para ser utilizada com pessoas idosas, objetivando aumento do desempenho de força (trabalho e potência) dos músculos extensores e flexores do joelho.

Palavras-chave: Trabalho; Envelhecimento; Treinamento.

Abstract - Step-training is associated with strength improvement of the lower limbs. Muscle strength is a critical component for the maintenance of functional capacity. The objective of the present study was to determine the effect of 6 weeks of step-training on work and power of the lower limbs in older women. Thirteen healthy and active women volunteered to participate in the study. All subjects underwent step-training classes three times per week for $60 \mathrm{~min}$. Strength variables of the knee extensor and flexor muscles were measured with a Biodex System 3 Pro isokinetic dynamometer. Muscle work and power were assessed at an angular velocity of 60 and $180 \%$ s. A significant difference $(p<0.05)$ in knee flexor muscle work was observed between pre-and post-test at 60 and $180 \%$. There was a significant difference $(p<0.05)$ in knee extensor muscle work between pre-and post-test at $60 \%$ significant differences were observed between pre-and post-test values of knee flexor muscle power at $60 \% \mathrm{~s}(p<0.05)$ and knee extensor muscle power at 60 and $180 \%$ ( $p<0.05)$. In conclusion, step-training can be recommended as an alternative physical activity to increase strength performance (work and power) of the knee extensor and flexor muscles in older subjects.

Key words: Work; Aging; Training. 


\section{INTRODUÇÃO}

O declínio na capacidade fisiológica dos seres humanos é uma consequência inevitável do processo de envelhecimento biológico'. Uma das alterações é a diminuição da capacidade de produzir força muscular ${ }^{2,3}$ em função, por exemplo, da redução da área de seção transversa do músculo ${ }^{4}$. Essa alteração dos níveis de força muscular pode limitar a capacidade de realização das atividades da vida diária e aumentar o risco de quedas ${ }^{5,6}$, o que leva à diminuição da autonomia motora e, consequentemente, da qualidade de vida das pessoas idosas? ${ }^{7}$. Como os extensores e flexores do joelho estabilizam a articulação, o declínio da força desses grupos musculares também reduz a capacidade de absorção de impactos, ou seja, diminui a capacidade em proteger a articulação contra cargas mecânicas ${ }^{8}$. Neste sentido, atividades que proporcionem um aumento da força muscular são recomendadas para idosos ${ }^{9}$, porque podem resultar em benefícios para o desempenho funcional ${ }^{10}$. Vários estudos investigaram o impacto de programas de treinamento com pesos em parâmetros da força muscular em idosos ${ }^{4,11,12}$ e no equilíbrio ${ }^{13}$. Diferentes autores relatam que o step-training pode ser usado como meio de treinamento para desenvolver a força muscular ${ }^{14,15}$. Entretanto, poucos estudos avaliaram o efeito do step-training em parâmetros da força muscular ${ }^{14}$ e resultados de pesquisas sobre o efeito desta atividade na resposta da força muscular de membros inferiores em pessoas idosas ainda não foram relatados. O fato de que o step-training é uma atividade caracterizada por subir e descer de uma plataforma de altura regulável, com ritmo variado e movimentos coreografados ${ }^{16}$, que submete os indivíduos a uma demanda moderada e repetida de força de reação no solo (1.95 a 2.32 vezes o peso corporal $^{15}$, espera-se que esta atividade represente um estímulo mecânico suficiente para provocar adaptações no sistema neuromuscular, que possibilitem um aumento da força muscular. A atividade eletromiográfica dos grupos musculares extensor e flexor do joelho aumenta, significativamente, quando a altura da plataforma é elevada ${ }^{17}$. Portanto, o estímulo de treinamento pode ser modulado por meio da alteração da altura de subida/descida. Desta forma, o objetivo do presente estudo foi investigar o efeito de um programa de seis semanas de step-training nos parâmetros trabalho e potência de membros inferiores, mensurados em aparelho isocinético, em mulheres ativas fisicamente acima de 60 anos de idade.

\section{PROCEDIMENTOS METODOLÓGICOS}

\section{Amostra}

A amostra foi composta por 55 mulheres ativas e saudáveis, que frequentavam aulas de ginástica para a terceira idade dentro do projeto de extensão oferecido pelo curso de Educação Física da Universidade Federal de Minas Gerais (UFMG). As voluntárias foram contatadas e receberam informações sobre a pesquisa. Os seguintes critérios de inclusão foram considerados, inicialmente, para seleção da amostra: idade igual ou superior a 60 anos, ausência de história de patologia ortopédica e reumatológica e tempo mínimo de prática de atividade física de seis meses. Após a aplicação desses critérios de inclusão, permaneceram 30 mulheres. Após a aplicação do critério de exclusão, disponibilidade para frequentar 3 sessões de treinamento semanal, um total de 17 voluntárias foram selecionadas. Durante a realização do projeto, duas voluntárias abandonaram o programa por motivos pessoais e duas por motivos de logística. Portanto, a amostra final foi composta por 13 voluntárias. Nenhuma das voluntárias havia participado, anteriormente, em programas de step-training e foram instruídas a não participarem de outros programas de atividade física durante a realização da pesquisa. A tabela 1 informa sobre as características antropométricas das voluntárias.

Tabela 1. Valores mínimos, máximos, média e desvio padrão das características antropométricas das voluntárias $(n=13)$.

\begin{tabular}{lllll}
\hline & Mínimo & Máximo & Média & d.p. \\
\hline Idade (anos) & 62,0 & 75,0 & 66,0 & $\pm 3,6$ \\
Massa corporal $(\mathrm{kg})$ & 55,0 & 78,2 & 65,3 & $\pm 7,6$ \\
Estatura $(\mathrm{cm})$ & 146,0 & 162,0 & 153,9 & $\pm 4,8$ \\
IMC $\left(\mathrm{kg} / \mathrm{cm}^{2}\right)$ & 22,7 & 33,0 & 27,6 & $\pm 3,1$ \\
\hline
\end{tabular}

IMC- índice de massa corporal, d.p. - desvio padrão

\section{Procedimentos}

Após aprovação do projeto pelo Comitê de Ética e Pesquisa da Universidade Federal de Minas Gerais, com parecer $n^{\circ} 39 / 02$, as voluntárias receberam informações detalhadas sobre os procedimentos da pesquisa e assinaram um termo de consentimento livre e esclarecido. Em seguida, foi realizado o registro das características antropométricas e uma sessão de familiarização com o dinamômetro isocinético para garantir o registro e posicionamento correto durante a avaliação. Após a sessão de familiarização, foi realizada a coleta de dados do pré-teste. O mesmo procedimento de coleta foi adotado no pós-teste, depois de um período de seis semanas de treinamento. 


\section{Teste Isocinético}

Os testes isocinéticos de extensão e flexão do joelho foram realizados no modo concêntrico-concêntrico, utilizando o dinamômetro isocinético Biodex System 3 Pro. Os testes foram realizados nas velocidades angulares de 60 e 180\%, que são recomendadas na realização de testes isocinéticos ${ }^{17}$ e foram utilizadas em idosos para mensurar a força muscular dos membros inferiores ${ }^{19}$, o que possibilitaria futuras comparações. O protocolo constituiu-se das seguintes etapas: a) Atividade preparatória: as voluntárias foram submetidas a um período de cinco minutos de atividade preparatória em um cicloergômetro (Marca Vitally), com a potência média de 25 watts, mantendo 60rpm. b) Posicionamento e alinhamento: as voluntárias foram posicionadas sentadas na cadeira do dinamômetro isocinético com o encosto da cadeira inclinado a $85^{\circ}$. A articulação do joelho foi alinhada com o eixo de rotação do dinamômetro na altura do epicôndilo lateral do fêmur. $\mathrm{O}$ braço de alavanca foi posicionado paralelamente à perna, com a almofada de apoio fixada no terço distal anterior da mesma, imediatamente acima do maléolo lateral. Todas as informações sobre este posicionamento no aparelho foram registradas para cada indivíduo com o objetivo de repeti-lo no pós-teste. c) Estabilização: durante a realização do teste, as voluntárias foram estabilizadas no tronco, pelve e no terço distal da coxa com a ajuda de cintos ajustáveis e também foram orientadas a segurar no suporte para as mãos. d) Correção do efeito da gravidade: antes da realização do teste, foram adotados os procedimentos de "correção do efeito da gravidade", segundo as instruções do fabricante contidas no manual do equipamento. e) Familiarização: para familiarização com o equipamento e com os procedimentos do teste, foram realizados previamente ao teste, três e cinco movimentos de flexão e extensão do joelho em esforços submáximos para as velocidades angulares de 60 e 180\%, respectivamente. f) Teste: após os procedimentos descritos, as voluntárias realizaram o teste nas velocidades angulares de 60 e $180 \%$ em esforço máximo. Foram realizadas cinco repetições a $60 \%$ s e 15 repetições a $180 \%$ s, sendo que um intervalo de 30 segundos foi mantido entre as etapas com as diferentes velocidades angulares. A amplitude de movimento total testada foi de $85^{\circ}$, iniciando do ângulo de $90^{\circ}$ de flexão de joelho. As voluntárias foram testadas sempre na mesma sequência, ou seja, $60 \%$ s seguido da velocidade angular de $180 \%$ s. O teste foi aplicado para o membro inferior dominante, sendo este definido como o membro inferior que o sujeito utilizaria para chutar uma bola. Foi feito o encorajamento verbal durante a realização do teste para que as idosas movessem a alavanca do dinamômetro com a maior força e o mais rápido possível. g) Variáveis: potência média e trabalho médio foram determinados como variáveis da força muscular. A diminuição do índice de queda em idosos é um aspecto central nas discussões, envolvendo a prescrição de diferentes intervenções. A redução da potência muscular é um indicador de déficit de equilíbrio e de risco de quedas ${ }^{13}$. De acordo com Salem et al. ${ }^{19}$, a capacidade de realizar trabalho explica 54\% da variância do desempenho em testes funcionais de alta intensidade, enquanto o pico de torque apenas $41 \%$.

\section{Protocolo do step-training}

Todas as voluntárias participavam de um programa regular de ginástica localizada, com duração de 60 minutos, em média, três vezes por semana, por um período mínimo de seis meses. As voluntárias então substituiram o programa usual de atividade física por um treinamento de seis semanas de steptraining, três vezes por semana $\left(2^{\mathrm{a}}, 4^{\mathrm{a}}\right.$ e $6^{\mathrm{a}}$-feira), com duração de 60 minutos cada sessão. Cada sessão de treinamento foi caracterizada por uma atividade preparatória de dez minutos, 40 minutos de step-training e dez minutos de "volta à calma". Por meio de sessões piloto de step-training com outros grupos de idosos, foram testados e selecionados os movimentos básicos do repertório de movimentos para os membros inferiores, tronco e membros superiores, assim como possíveis combinações coreográficas. Os seguintes movimentos fizeram parte da rotina: passo básico, V step, L step, movimentos laterais (elevação de joelho, chute lateral, flexão de joelho, extensão de quadril), repetidores, movimentos de membros superiores (flexão de cotovelo). Esses movimentos foram sempre realizados no início da rotina de cada sessão de treinamento. Nas duas primeiras semanas, foram combinados os exercícios mais simples (por ex.: passo básico, flexão de joelho) e nas outras quatro semanas, as combinações mais complexas (por ex.: V step e repetidores). $\mathrm{O}$ aumento na intensidade do estímulo durante o programa de treinamento foi realizado por meio do aumento na cadência musical e altura da plataforma. Na primeira semana, a cadência era de 120 bpm (batimentos por minuto) e nas demais semanas a cadência iniciava com 120 bpm e terminava com 126 bpm. As voluntárias receberam plataformas de dez centímetros de altura, durante as três primeiras semanas de treinamento e plataformas de $15 \mathrm{~cm}$ de altura, nas últimas três semanas 
do programa de treinamento.

\section{Análise estatística}

Após uma análise descritiva, foi realizado o teste Kolmogorov-Smirnov, que indicou uma distribuição normal dos dados, e o teste de Levené, mostrando que os dados apresentavam homogeneidade. Para verificar as diferenças entre as médias do pré e pós-teste, foi utilizado um teste T pareado. O nível de significância estabelecido foi de $\mathrm{p}<0,05$ e o pacote Statistica versão 7.0 foi usado para a execução dos cálculos.

\section{RESULTADOS}

\section{Trabalho}

A tabela 2 apresenta a média e o desvio padrão do parâmetro trabalho relativo às musculaturas extensora e flexora do joelho.

Na comparação das médias do trabalho entre o pré e o pós-teste para as velocidades angulares de 60 e 180\%, o teste T pareado mostrou que o trabalho no pós-teste aumentou significativamente para a musculatura extensora do joelho somente na velocidade angular de $60 \% \mathrm{~s}(\mathrm{p}=0,027)$. O resultado do teste $T$ pareado indicou que o trabalho para a musculatura flexora do joelho aumentou significativamente em ambas as velocidades angulares testadas $(60 \%-p=0,001 ; 180 \%-p=0,044)$.

\section{Potência}

A tabela 3 apresenta os valores médios e os desvios padrão do parâmetro potência, envolvendo as mus- culaturas extensora e flexora do joelho.

Os resultados mostraram que a potência no pós-teste aumentou significativamente em ambas as velocidades angulares para a musculatura extensora do joelho $(60 \% \mathrm{~s}-\mathrm{p}=0,002 ; 180 \% \mathrm{~s}-\mathrm{p}=$ $0,006)$. Para a musculatura flexora do joelho, foi verificada diferença significativa, comparando pré e pós-teste somente na velocidade angular de $60 \%$ $(p=0,004)$.

\section{DISCUSSÃO}

No grupo experimental, foram observados aumentos significativos nos valores do trabalho para a musculatura extensora e flexora do joelho nas velocidades angulares de $60 \%$ s e 60 e $180 \%$, respectivamente. Estes resultados mostram que esses grupos musculares aumentaram a capacidade de produção de torque durante o movimento articular. $\mathrm{O}$ aumento do trabalho pode estar relacionado com uma capacidade de produzir maiores valores de torque, durante o movimento articular, nas velocidades de 60 e 180\% ou com a manutenção da produção de torque no decorrer da amplitude de movimento (ADM) testada durante as cinco repetições. Essa manutenção pode ser representada por uma menor redução do torque médio verificado nas primeiras curvas torque x ADM, em comparação com o rendimento de torque médio, nas últimas repetições do protocolo de testes, nas velocidades de 60 e $180 \%$ s. Ambas as explicações podem possibilitar um maior torque médio durante o protocolo de teste. Essas

Tabela 2. Média e desvio padrão (entre parênteses) do trabalho relativo à musculatura extensora e flexora do joelho a 60 e $180 \%$ na condição pré e pós-teste.

\begin{tabular}{|c|c|c|c|c|c|c|c|c|}
\hline \multirow{4}{*}{ Grupo } & \multicolumn{8}{|c|}{ Trabalho (J) } \\
\hline & \multicolumn{4}{|c|}{ Extensão } & \multicolumn{4}{|c|}{ Flexão } \\
\hline & \multicolumn{2}{|c|}{$60 \% \mathrm{~s}$} & \multicolumn{2}{|c|}{$180 \% \mathrm{~s}$} & \multicolumn{2}{|c|}{$60 \% \mathrm{~s}$} & \multicolumn{2}{|c|}{$180 \% \mathrm{~s}$} \\
\hline & Pré & Pós & Pré & Pós & Pré & Pós & Pré & Pós \\
\hline \multirow{2}{*}{ EXP } & 458,6 & $497,5^{*}$ & 783,3 & 813,8 & 241,5 & $283,0^{*}$ & 439,6 & $476,0^{*}$ \\
\hline & $( \pm 121,1)$ & $( \pm 109,0)$ & $( \pm 175,3)$ & $( \pm 184,0)$ & $( \pm 76,6)$ & $( \pm 68,5)$ & $( \pm 177,7)$ & $( \pm 165,7)$ \\
\hline
\end{tabular}

* p<0,05 (comparação pré x pós); J = joule

Tabela 3. Média e desvio padrão (entre parênteses) da potência relativa à musculatura extensora e flexora do joelho a 60 e $180^{\circ} / \mathrm{s}$ na condição pré e pós-teste.

\begin{tabular}{|c|c|c|c|c|c|c|c|c|}
\hline \multirow{4}{*}{ Grupo } & \multicolumn{8}{|c|}{ Potência (W) } \\
\hline & \multicolumn{4}{|c|}{ Extensão } & \multicolumn{4}{|c|}{ Flexão } \\
\hline & \multicolumn{2}{|c|}{$60 \% \mathrm{~s}$} & \multicolumn{2}{|c|}{$180 \% \mathrm{~s}$} & \multicolumn{2}{|c|}{$60 \% \mathrm{~s}$} & \multicolumn{2}{|c|}{$180 \% \mathrm{~s}$} \\
\hline & Pré & Pós & Pré & Pós & Pré & Pós & Pré & Pós \\
\hline \multirow{2}{*}{ EXP } & 62,1 & $66,8^{*}$ & 85,9 & $90,5^{*}$ & 33,7 & $38,7^{*}$ & 49,0 & 53,7 \\
\hline & $( \pm 16,4)$ & $( \pm 14,8)$ & $( \pm 21,0)$ & $( \pm 20,4)$ & $( \pm 11,2)$ & $( \pm 9,3)$ & $( \pm 22,7)$ & $( \pm 20,4)$ \\
\hline
\end{tabular}

* p <0,05 (comparação pré $\mathrm{x}$ pós); $\mathrm{W}=$ watts 
alterações verificadas podem estar associadas às características da demanda física impostas pelo step-training, uma vez que a variação da medida relacionada com o procedimento de testagem com aparelhos isocinéticos é muita baixa. Recentemente, Hartman et al. ${ }^{20}$ investigaram a confiabilidade das medidas realizadas em aparelho isocinético, idêntico ao utilizado no presente estudo e com indivíduos idosos. Esses autores concluíram que este aparelho pode ser utilizado com segurança para determinar os efeitos de programas de atividades.

Müller et al. ${ }^{21}$ concluíram que os grupos musculares quadríceps e isquiotibiais apresentam maior ativação eletromiográfica comparados com o glúteo máximo, reto abdominal e eretores da espinha durante a atividade de step-training. Levando em consideração esses resultados, esperava-se um aumento significativo na força produzida por estes grupos musculares, que são os principais músculos extensores e flexores do joelho, após sessões de step-training. De acordo com Anderson \& Kearney $y^{22}$ o aumento da força muscular induzido pelo exercício físico está relacionado, entre outros, com a quantidade de tensão produzida pela musculatura envolvida, onde os aumentos da força muscular resultam da elevação progressiva do nível de tensão. No presente estudo, três manipulações foram realizadas para aumentar a exigência fisiológica na sessão de treinamento: o aumento na cadência musical (120 a 126 batimentos por minuto), a alteração na altura das plataformas de 10 para $15 \mathrm{~cm}$ e o aumento na frequência de alguns movimentos (por ex.: repetidores) com maior demanda fisiológica ${ }^{23} \mathrm{e}$ mecânica ${ }^{15}$, no decorrer das sessões. Essa carga de treinamento crescente foi suficiente para estimular as musculaturas envolvidas e aumentar a capacidade das mesmas em produzir maiores torques.

No presente estudo, o aumento na capacidade de realizar trabalho pelas musculaturas extensora e flexora do joelho pode ser explicado pela alteração de mecanismos neurais. As adaptações neurais são descritas como a principal causa de incrementos na produção de força, nas primeiras semanas de treinamento, enquanto que o aumento na área transversal do músculo é considerado a principal causa da maior produção de força após períodos mais longos de treinamento ${ }^{24}$. A comprovação de ganhos de força em virtude de adaptações neurais pode ser verificada por meio da análise do sinal eletromiográfico. Embora neste estudo a investigação de variáveis eletromiográficas não tenha sido o foco, acredita-se que o aumento da capacidade de produzir trabalho seja decorrente, principalmente, de adaptações neurais, caracterizadas pelo aumento no recrutamento e/ou na sincronização na ativação de unidades motoras de diferentes músculos devido à duração do programa de treinamento ter sido de seis semanas ${ }^{23}$. Portanto, estudos futuros deverão ser conduzidos para verificar a hipótese de que o steptraining influencia significativamente os parâmetros da força muscular e do sinal eletromiográfico dos músculos exercitados.

Na presente pesquisa, vários aspectos relacionados com a progressão da carga de treinamento foram estabelecidos e aplicados ao longo das sessões de treinamento. Isto pode ter sido o fator determinante para o aumento do parâmetro trabalho verificado neste estudo, diferentemente dos resultados verificados na pesquisa de Koenig et al. ${ }^{14}$. No estudo realizado por Koenig et al. ${ }^{14}$, objetivou-se investigar alterações no pico de torque, na potência e na resistência de força dos músculos quadríceps e isquiotibiais após um programa de dez semanas de step-training. A resistência muscular (soma total do trabalho para 20 repetições a $300 \%$ s) e a potência não apresentaram diferença significativa para nenhum dos dois grupos musculares analisados na comparação pré e pós-teste. Esses resultados foram explicados pelos autores pela ausência de manipulações na progressão da carga de treinamento, uma vez que a altura da plataforma e a cadência musical foram mantidas as mesmas durante o período de treinamento. Esta argumentação reforça as justificativas mencionadas acima para o aumento significativo do parâmetro trabalho verificado no presente estudo.

Partindo da análise dos resultados da variável trabalho e da progressão da carga de treinamento executada durante o período de treinamento, na presente pesquisa, foram esperadas alterações significativas também na variável potência. Os resultados indicam que a potência foi alterada após seis semanas de step-training, nas velocidades angulares de 60 e $180 \%$ e $60 \%$, para as musculaturas extensora e flexora do joelho respectivamente, contradizendo os dados apresentados por Koenig et al..$^{14}$.

Uma possível explicação para este resultado pode estar relacionada com os exercícios realizados na rotina do step-training. $\mathrm{O}$ exercício de flexão de joelho era executado em todas as sessões de treinamento e é caracterizado por um exercício em cadeia cinética aberta. Neste sentido, um teste que apresenta características similares àquelas da tarefa motora treinada é mais sensível para detectar as alterações provocadas pelo programa de treinamento ${ }^{25}$. Deve-se considerar, também, que a adaptação é específica à velocidade do movimento 
utilizada nos treinamentos ${ }^{26,27}$, desta forma, a baixa cadência musical utilizada no presente estudo pode representar um padrão de movimento caracterizado por uma execução mais lenta. Este padrão de exigência poderia se aproximar mais da velocidade angular de $60 \%$ do que $180 \%$, o que explicaria a não alteração da potência a $180 \%$ s.

Como o step-training requer que o indivíduo realize deslocamentos da massa corporal ,alternando constantemente o membro inferior de apoio dentro de uma frequência musical, movimentos laterais e coreografados que exigem equilíbrio e agilidade, é possível hipotetizar que esta atividade tenha uma influência positiva sobre aspectos da aptidão física importante para idosos ${ }^{28}$. Neste sentido, seria interessante a realização de estudos futuros que investiguem se este tipo de atividade tem efeito em outros parâmetros importantes da aptidão física do idoso, como o equilíbrio, agilidade e desempenho em testes funcionais.

O delineamento quasi-experimental do tipo pré x pós-teste de um grupo foi utilizado ${ }^{29}$. A ausência de um grupo controle é uma limitação do presente estudo. Contudo, a decisão por este delineamento foi baseada na boa confiabilidade da mensuração, utilizando aparelhos isocinéticos ${ }^{20}$. Estas informações mostram que a influência da variação das medidas associadas ao aparelho isocinético é conhecida e por isso, não seria necessária a utilização de um grupo controle somente para esta finalidade. A presença de um grupo controle somente seria justificada se o objetivo fosse controlar o efeito do treinamento, uma vez que o grupo era ativo fisicamente e as adaptações específicas do programa de treinamento que estava sendo realizado, antes de iniciar o programa de step-training, poderiam apresentar um processo de reversibilidade. Isto poderia ser um fator de confusão em relação ao desempenho mensurado ao final do programa de step-training. Desta forma, para controlar esta situação experimental, o mais adequado seria que os indivíduos do grupo controle fossem indivíduos com características semelhantes à dos indivíduos do $\mathrm{Grupo}_{\text {trein }}$. Contudo, os indivíduos deveriam encerrar as atividades físicas realizadas no programa, o que não seria uma decisão adequada, uma vez que uma interrupção iria provocar uma redução nos benefícios associados à prática de atividade física regular daqueles indivíduos.

\section{CONCLUSÃO}

Os resultados deste estudo mostram que seis semanas de step-training conduziram para aumentos significativos da capacidade de realizar trabalho nos músculos extensores e flexores do joelho de pessoas idosas, na velocidade angular de $60 \%$. Os resultados reforçam, ainda, a premissa de que os idosos podem ser engajados em programas de treinamento com o step-training, podendo resultar em aumentos significativos, também, na potência muscular de membros inferiores. Isto indica que o step-training pode ser mais uma alternativa de atividade a ser utilizada com pessoas idosas.

\section{REFERÊNCIAS BIBLIOGRÁFICAS}

1. Lemura LM, Von duvillard SP, Mookerjee S. The effects of physical training on functional capacity in adults. J Sports Med Phys Fitness 2000;40(1):1-10.

2. Akima H, Kano Y, Enomoto Y, Ishizu M, Okada M, Oishi Y, et al. Muscle function in 164 men and women aged 20-84 yr. Med Sci Sports Exerc 2001;33(2):220-226.

3. Lindle RS, Metter EJ, Lynch NA, Fleg JL, Fozard JL, Tobin J, et al. Age and gender comparisons of muscle strength in 654 women and men aged 20-93yr. J Appl Physiol 1997;83(5):1581-1587.

4. Frontera WR, Meredith CN, O'reilly KP, Knuttgen HG, Evans WJ. Strength conditioning in older men skeletal muscle hypertrophy and improved function. J Appl Physiol 1988;64(3):1038-1044.

5. Snow CM. Exercise effects on falls in frail elderly: focus on strength. J Appl Biomech 1999(1):15:84-91.

6. Robinovitch SN, Hsiao ET, Sandler R, Cortez J, Liu Q, Paiement GD. Prevention of falls and fall-related fractures through biomechanics. Exerc Sport Sci Rev 2000;8(1):74-79.

7. Beissner KL, Collins JE, Holmes H. Muscle force and range of motion as predictors of function in older adults. Phys Ther 2000;80(6):556-563.

8. Radin EL, Yang KH, Riegger C, Kish VL, O'Connor JJ. Relationship between lower limb dynamics and knee joint pain. J Orthop Res 1991;9(3):398-405.

9. American College of Sports Medicine-ACSM. Position stand on exercise and physical activity for older adults. Med Sci Sports Exerc 1998;30(6):992-1008.

10. Chandler JM, Duncan PW, Kochersberger G, Studenski $\mathrm{S}$. Is lower extremity strength gain associated with improvement in physical performance and disability in frail, community-dwelling elders? Arch Phys Med Rehabil 1998;79(1):24-30.

11. Häkkinen K, Kraemer WJ, Newton RU, Alen M. Changes in eletromyographic activity, muscle fiber and force production characteristics during heavy resistance/ power strength training in middle-aged and older men and women. Acta Physiol Scand 2001;171(1):51-62.

12. Andersen LL, Andersen JL, Magnusson P, Suetta C, Madsen JL, Christensen LR, et al. Changes in the human muscle force-velocity relationship in response to resistance training and subsequent detraining. J Appl Physiol 2005;99(1):87-94. 
13. Orr R, Vos NJ, Singh Na, Ross DA, Stavrinos TM, Fiatarone $\mathrm{M}$. Power training improves balance in healthy older adults. J Gerontology 2006;61A(1):78-85.

14. Koenig JM, Jahn DM, Dohmeier TE, Cleland JW. The effect of bench step aerobics on muscular strength, power and endurance. J Strength Cond Res 1995;9(1):43-46.

15. Dyson RJ, Farrington TA. Step aerobics vertical ground reaction force and exercise duration. J Hum Mov Stud 1995;29(1):79-87.

16. Scharff-Olson M, Williford HN, Blessing DL, Brown JA. The physiological effects of bench/step exercise. Sports Med 1996;21(3):164-175.

17. Franco S, Santos R, Pezarat-Corria P, Veloso A. Influence of bench heigth on muscle participation pattern in step exercise (Abstract). V Annual Congress of the European College of Sport Science. Jyvaskyla: 2000, p.269.

18. Aquino MA, Leme LEG, Amatuzzi MM, Greve JMDA, Terreri ASAP, Andrusaitis FR, et al. Isokinetic assessment of knee flexor/extensor muscular strength in elderly women. Rev Hosp Clín Fac Med S Paulo 2002; 57(4):131-134.

19. Salem GJ, Wang MY, Young JT, Marion M, Greendale GA. Knee strength and lower- and higher-intensity functional performance in older adults. Med Sci Sports Exerc 2000; 32(10):1679-1684.

20. Hartmann A, Knols R, Murer K, de Bruin ED. Reproducibility of an isokinetic strength-testing protocol of the knee and ankle in older adults. Gerontology 2009;55(3):259-268.

21. Müller T, Bezerra AF, Barrivieira AC, Zanchetta AM, Gonçalves M. Análise eletromiográfica durante o movimento do step. VI Congresso Brasileiro de Biomecânica. Brasília: 1995, p.238-243.
22. Anderson T, Kearney J. Effects of three resistance training programs on muscular strength and absolute and relative endurance. Res Q Exerc Sport 1982; 53(1):1-7.

23. Olson MS, Williford HN, Blessing DL, Greathouse R. The cardiovascular and metabolic effects of bench stepping exercise in females. Med Sci Sports Exerc 1991;23(11):1311-1317.

24. Schmidtbleicher D. Training for power events. In: Komi PV, editor. Strength and power in sport, London: Blackwell Science; 1992. p.381-395.

25. Brown LE, Weir JP. ASEP procedures recommendation I: accurate assessment of muscular strength and power. J Exerc Phys 2001;4(1):1-21.

26. Behm DG, Sale DG Velocity specificity of resistance training. Sports Med 1993; 15(6):374-388.

27. Aagaard P, Simonsen EB, Trolle M, Bangsbo J, Klausen K. Specificity of training velocity and training load on gains in isokinetic knee joint strength. Acta Physiol Scand 1996;156(2):123-129.

28. Rogers MW, Johnson ME, Martinez KM, Mille ML, Hedman LD. Step-training improves the speed of voluntary step initiation in aging. J Gerontol A Biol Sci Med Sci 2003;58A(1):46-51.

29. Portney LG, Watkins MP. Foundations of clinical research: applications to practice. Upper Saddle River: Prentice Hall Health; 2000.

\section{Endereço para correspondência \\ Daniela Coelho Zazá}

R. Alsácia, 124/402B, Minas Brasil. 30730-140 - Belo Horizonte,MG. Brasil.

E-mail: zazadaniela@hotmail.com 